\title{
Collective cell migration of epithelial cells driven by chiral torque generation
}

\author{
Takaki Yamamoto $\odot,{ }^{1,2, *}$ Tetsuya Hiraiwa $\odot,{ }^{3,4}$ and Tatsuo Shibata $\oplus^{1,+}$ \\ ${ }^{1}$ Laboratory for Physical Biology, RIKEN Center for Biosystems Dynamics Research, Kobe 650-0047, Japan \\ ${ }^{2}$ Nonequilibrium Physics of Living Matter RIKEN Hakubi Research Team, RIKEN Center for Biosystems Dynamics Research, \\ 2-2-3 Minatojima-minamimachi, Chuo-ku, Kobe 650-0047, Japan \\ ${ }^{3}$ Mechanobiology Institute, National University of Singapore, Singapore 117411, Singapore \\ ${ }^{4}$ Universal Biology Institute, University of Tokyo, 7-3-1 Hongo Bunkyo-ku, Tokyo 113-0033, Japan
}

(Received 6 September 2019; accepted 12 November 2020; published 7 December 2020)

\begin{abstract}
Various multicellular tissues show chiral morphology. Experimental studies have suggested that this can originate from the chirality of individual cells. However, no theory has been proposed to connect the cellular chiral torque and multicellular chiral morphogenesis. We propose a model of confluent tissue dynamics with cellular chiral torque. We found that cells migrate unidirectionally under a gradient of cellular chiral torque. While the migration speed varies depending on the tissue's mechanical parameters, it is determined solely by a structural order parameter for the liquid-to-solid transition in confluent tissues.
\end{abstract}

DOI: 10.1103/PhysRevResearch.2.043326

\section{INTRODUCTION}

The establishment of left-right (LR) asymmetry in tissues and organs is an intriguing event in development, which involves the coordinated activity of cells and molecules [1-4]. Since proteins as the basic components of biological systems are chiral molecules, the breaking of LR symmetry can be a collective property orchestrated by these chiral molecular components [5,6].

For the LR asymmetry in tissue morphogenesis, it has been shown that in Drosophila, the embryonic hindgut twists unidirectionally [7], and the male genitalia undergo a clockwise rotation when viewed from the apical side [8,9]. These are epithelial tissues, which consist of epithelial cells that adhere to each other through cell-cell junctions [10]. During the hindgut twisting, the hindgut epithelial cells exhibit chiral properties in their shape and other properties [7]. In the genitalia rotation, the surrounding epithelial cells move collectively in the clockwise direction driven by LR asymmetric positional rearrangements of cells, which rotates the genital disk $[9,11]$. Even more generally, such chiral cell rearrangements can induce unidirectional cell migration within an epithelial tissue [12]. The above examples suggest that a collective behavior of cellular chirality gives rise to the tissue LR asymmetry.

At the single-cell level, several types of cells have been reported to exhibit chiral dynamics. Examples include the extension of neurites in cultured nerve cell [13], the chiral

\footnotetext{
*takaki.yamamoto@ riken.jp

†tatsuo.shibata@ riken.jp

Published by the American Physical Society under the terms of the Creative Commons Attribution 4.0 International license. Further distribution of this work must maintain attribution to the author(s) and the published article's title, journal citation, and DOI.
}

movement of $C$. elegans cell cortex [14], nuclear rotation in melanophores of zebrafish [15], and actin cytoskeleton swirling in human foreskin fibroblasts (HFFs) cultured on a micropattern [16]. Remarkably, in zebrafish melanophores and the HFFs on the micropattern, the single cells can cellautonomously generate chiral torques in such a way that the apical side (top side) of cells exhibits rotations with respect to the basal side (bottom side) which adheres to substrates. Although a variety of specific mechanisms generate chiral torque at the cellular level, many of these chiral properties are governed by active chiral processes of actomyosin cytoskeleton [17-19].

Since actomyosin is a ubiquitous component of eukaryotic cells, it is natural to consider that chiral torque generation is not a feature restricted to the specific cells mentioned above. In particular, we consider a situation where chiral torque is generated by individual single epithelial cells in a tissue, motivated by the LR symmetry breaking of the epithelial tissues [7-9], and the chiral torque generation of single cells [13-16]. Based on the cell vertex model (CVM) [20], we propose a theoretical model of dynamics of an epithelial tissue with a chiral torque generated by individual single cells. Then, we investigate the tissue scale dynamical emergent properties.

\section{MODEL DESCRIPTION}

To derive our model, we start with investigating how the chiral torque generation determines the rotational motion of apical and basal sides of an isolated single cell. Let us consider a situation where a single cell is put on a frictional rigid substrate as in Fig. 1(a). We represent the apical and basal sides as two rotating rigid disks driven by a torque dipole consisting of two torque monopoles $\vec{\tau}_{m}=\tau_{m} \vec{n}$ and $-\vec{\tau}_{m}$, respectively, as schematically shown in Fig. 1(a). The torque dipole is considered to be generated by actomyosin $[17,18]$. Here, $\vec{n}$ is a unit normal vector from the basal to apical sides. Let 
(a)

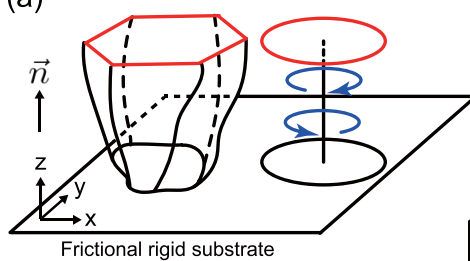

(b)

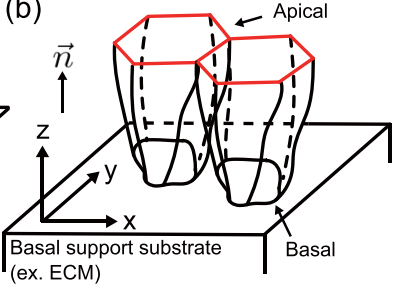

(c)

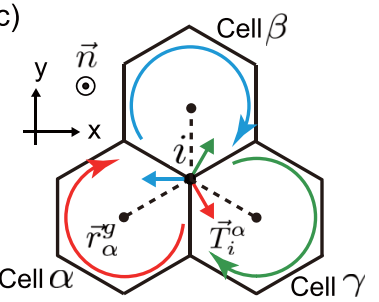

(d)

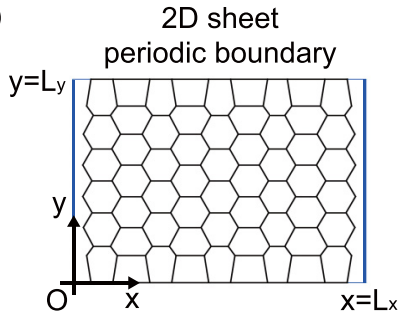

FIG. 1. (a) Schematic of the disk-shaft model of a rotating cell on a frictional rigid substrate. The sense of a torque dipole driving the cellular rotation is represented by curved arrows. In the model, the apical and basal sides are modeled as red and black rotating disks, respectively. (b) Schematic of an epithelial tissue on a basal support substrate. (c) Schematic of the torque forces exerted on the $i$ th vertex by the surrounding three cells generating chiral torque. The curved and solid arrows represent the chiral torque generation and torque forces, respectively, for positive $v_{\alpha}$. (d) $2 \mathrm{D}$ cellular sheet with the periodic boundary condition in the $x$ direction.

$\vec{\omega}_{a}=\omega_{a} \vec{n}$ and $\vec{\omega}_{b}=\omega_{b} \vec{n}$ be the angular velocity of the apical and basal sides, respectively. We consider a coupling between the rotation of the basal and apical disks via rotational viscous dissipation inside the cell which comes from the difference in the angular velocities $\left(\omega_{a}-\omega_{b}\right) \vec{n}$. We here consider the rotational degrees of freedom around the axis $\vec{n}$. The torque balance equations for the apical and basal sides are given by

$$
\begin{gathered}
\gamma_{c}\left(\omega_{a}-\omega_{b}\right)=\tau_{m}, \\
\gamma_{c}\left(\omega_{b}-\omega_{a}\right)+\eta_{s} \omega_{b}=-\tau_{m},
\end{gathered}
$$

respectively. Here, $\gamma_{c}$ and $\eta_{s}$ are the rotational viscous coefficient of the cell and the rotational friction coefficient between the cell and the substrate, respectively. We call this simple model as the "disk-shaft model." Solving the equations for $\eta_{s} \neq 0$, we readily obtain $\omega_{b}=0$ and $\gamma_{c} \omega_{a}=\tau_{m}$. Hence, this model explains the continuously rotating apical surface of the single cell reported in $[15,16]$. Note that we neglected the twist elasticity of the cell since the continuously rotating apical surface is realized only when the twist viscosity is dominant over the twist elasticity. The disk-shaft model shows that the torque dipole results in the chiral toque $\vec{\tau}_{m}$ on the apical side of the cell since the basal disk is fixed due to the friction from the substrate.

We integrate this insight into the model of epithelial tissue. Dynamics of epithelial cell monolayer is often described by a two-dimensional (2D) CVM, in which the cells are described by polygons with vertices and edges. In Fig. 1(b), we depict the three-dimensional (3D) structure of an epithelial tissue on the supporting substrate such as an ECM. We here consider a rigid frictional substrate for simplicity. At the apical side, the cells are tied to each other via the adherens junction, while the basal membrane of the cells is attached to the substrate. The edges of 2D CVM represent the adherens junction at the apical side which is mechanically supported by the actomyosin belt, while the basal side is assumed to be relatively mechanically independent between neighboring cells [Fig. 1(b)]. In 2D CVM, for the position of the $i$ th vertex $\vec{r}_{i}$, the mechanical properties at the apical side are described by a potential function $E\left(\left\{\vec{r}_{i}\right\}\right)$ in Eq. (3), and the force acting on each vertex is given by the derivative $-\partial E\left(\left\{\vec{r}_{i}\right\}\right) / \partial \vec{r}_{i}$ :

$$
\begin{aligned}
E\left(\left\{\vec{r}_{i}\right\}\right)= & \frac{K}{2} \sum_{\alpha=1}^{N}\left(A_{\alpha}-A_{0}\right)^{2}+\frac{K_{p}}{2} \sum_{\alpha=1}^{N}\left(P_{\alpha}-P_{0}\right)^{2} \\
& +\sum_{\langle i, j\rangle} \Delta \Lambda_{i j}(t) \ell_{i j} .
\end{aligned}
$$

Here, the first term on the right-hand side describes the area elasticity with the area $A_{\alpha}$ of cell $\alpha$, the elastic modulus $K$, and the preferred area $A_{0}$. The second term defines the perimeter elasticity with the perimeter length $P_{\alpha}$ of cell $\alpha$, the elastic modulus $K_{p}$, and the preferred perimeter length $P_{0}$. We assume for simplicity that these cellular mechanical properties $K, K_{p}, A_{0}, P_{0}$ are spatially homogeneous. $N$ is the total number of cells. The third term represents line tension with a magnitude $\Delta \Lambda_{i j}(t)$. Here, $\ell_{i j}$ is the length of a cell edge between $i$ th and $j$ th vertices.

In the disk-shaft model, we have shown that a single cell generates chiral torque $\vec{\tau}_{m}^{\alpha}$ at the apical side, which drives the chiral cellular rotation. To represent such a torque in the framework of the 2D CVM, we introduce the torque force around the cell center as shown in Fig. 1(c). The simplest form of the torque force on the $i$ th vertex exerted by cell $\alpha$ may be given by

$$
\vec{T}_{i}^{\alpha}=v_{\alpha}\left(\vec{r}_{i}-\vec{r}_{g}^{\alpha}\right) \times \vec{n},
$$

where $v_{\alpha}$ is the coefficient of the torque force generated by the cell $\alpha, \vec{r}_{g}^{\alpha}$ is the area centroid of cell $\alpha$ (see Appendix A for the definition of area centroid), and $\vec{n}$ is a unit normal vector from the basal to apical sides. See Appendix B for the detailed derivation of Eq. (4). The net torque force $\vec{T}_{i}$ on the $i$ th vertex can be represented by sum of the torque forces exerted by the surrounding cells $(\alpha, \beta, \gamma)$ :

$$
\vec{T}_{i}=\vec{T}_{i}^{\alpha}+\vec{T}_{i}^{\beta}+\vec{T}_{i}^{\gamma}
$$

The time-evolution equation for $\vec{r}_{i}$ is obtained by considering the force balance between the frictional force $\eta d \vec{r}_{i} / d t$ with friction constant $\eta$, the potential force $-\partial E\left(\left\{\vec{r}_{i}\right\}\right) / \partial \vec{r}_{i}$ derived from Eq. (3), and the torque force $\vec{T}_{i}$ given by Eq. (5) as follows:

$$
\eta \frac{d \vec{r}_{i}}{d t}=-\frac{\partial E\left(\left\{\vec{r}_{i}\right\}\right)}{\partial \vec{r}_{i}}+\vec{T}_{i}
$$

Here, the friction is caused by the viscous property of the tissue and the dissipation to the basal substrate. We introduce the friction force in a conventional way by assuming that the friction constant $\eta$ is spatially homogeneous, unless otherwise noted [9,11,12,21] (see also Appendix B). When the length of a cell edge falls below a threshold $l_{\text {th }}=0.03$ during a time evolution according to Eq. (6), a T1 transition is performed by flipping the edge by $90^{\circ}$ [20]. 
In what follows, we analyze Eqs. (3)-(6) with the following settings. We introduce a fluctuating tension $\Delta \Lambda_{i j}(t)$, whose dynamics is described by

$$
\frac{d \Delta \Lambda_{i j}(t)}{d t}=-\frac{\Delta \Lambda_{i j}(t)}{\tau}+\xi_{i j}(t),
$$

where $\left\langle\xi_{i j}(t)\right\rangle=0$ and $\left\langle\xi_{i j}\left(t_{1}\right) \xi_{k l}\left(t_{2}\right)\right\rangle=\delta_{i k} \delta_{j l} \delta\left(t_{1}-t_{2}\right)$. Here, $\Delta \Lambda_{i j}(t)$ satisfies $\left\langle\Delta \Lambda_{i j}(t)\right\rangle=0$ and $\left\langle\Delta \Lambda_{i j}\left(t_{1}\right) \Delta \Lambda_{k l}\left(t_{2}\right)\right\rangle=$ $\delta_{i k} \delta_{j l} \sigma^{2} e^{-\left|t_{1}-t_{2}\right| / \tau}$. Such time-correlated fluctuation of line tension is reported in [21]. We set $\tau=1$ in this paper for simplicity. Hereafter, we choose the units of length and forces such that the elastic modulus $K_{p}$ and the preferred cell area $A_{0}$ are unity, i.e., $K_{p}=1$ and $A_{0}=1$. With these units, $P_{0}$ is the so-called target shape index, which is a ratio between perimeter and square root of area $[22,23]$. If a single cell tends to be circular, $P_{0}=2 \sqrt{\pi} \sim 3.54$. We set $P_{0}=3.54$ and $K=10$ to avoid large cellular shape deformation unless otherwise noted. We consider a simple configuration of a rectangular epithelial sheet with size $L_{x}$ and $L_{y}$ in the $x$ and $y$ direction, respectively, as shown in Fig. 1(d). For the $x$ direction, we apply the periodic boundary condition at $x=0$ and $L_{x}$. The rectangular area size is equal to the number of cells so that the average cell area is set to be $\bar{A}_{\alpha}=1$. This configuration corresponds to the tube geometry, a biologically ubiquitous structure of organs, which exhibits chiral morphogenesis as twisting of a heart tube and hindgut. We simply consider that the cells are attached to the tissue boundary. We hence fix the $y$ coordinates of vertices on the bottom and top boundaries to $y=0$ and $L_{y}$, respectively. $\eta$ is set to 1 both at the bulk and the boundary $\left(y=0, L_{y}\right)$ unless otherwise noted. We calculate the time-evolution equation (6) with the time step $\Delta t=0.01$ using the forward Euler's method. We prepare initial cellular configurations packed with regular hexagonal cells to calculate the dynamics with $\sigma=0$ and $v_{\alpha}=0$ to relax the system, and then set $\sigma$ and $v_{\alpha}$ to the target values (see Appendix C). The numbers of cells in the initial hexagonal configuration in the $x$ and $y$ directions are, respectively, denoted as $N_{x}$ and $N_{y}$, and we set $N_{x}=10$ for all the simulations.

\section{RESULTS}

We first investigated the role of chiral torque generation on the dynamics of cells when the strength $v_{\alpha}$ of chiral torque generation is spatially homogeneous. As shown in Fig. 2(a), we found that the cells migrate bidirectionally when the fluctuation in line tension is present $\left(v_{\alpha}=0.2, \sigma=0.3, N_{y}=\right.$ 6 ). With negative $v_{\alpha}$, the direction of the cellular migration is reversed, confirming that the chiral torque generation is the driving force of the cellular migration. For sufficiently large system $\left(N_{y}=40\right)$, the flow is localized at the boundary and the bulk velocity vanishes [Fig. 2(b)]. This indicates that the torque force and the potential force are balanced in the bulk, while not balanced near the boundary. On the top and bottom boundaries, the torque forces on the vertices are exerted in the rightward and leftward directions, respectively, as depicted in Fig. 2(c), leading to the bidirectional cellular flow at the boundaries.

In the bulk, to understand how the torque force and the potential force are balanced, we consider a mean-field model where the torque force is exerted on a vertex $O$ surrounded by (a)

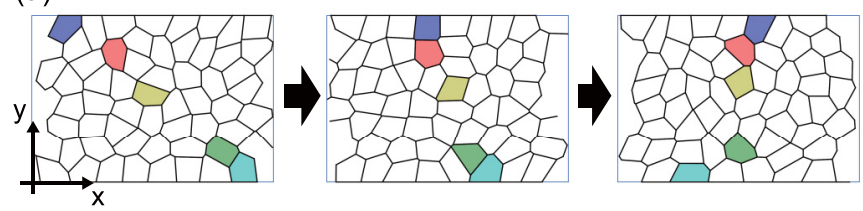

(b)

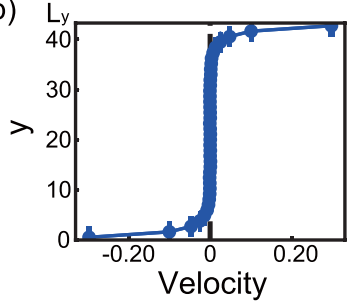

(c)

Torque force on the boundary

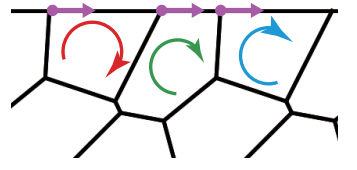

FIG. 2. (a) Time evolution of a cellular configuration in a numerical simulation ( $\left.v_{\alpha}=0.2, \sigma=0.3, N_{y}=6\right)$. The time interval between each configuration is 40 time units. The Supplemental Material S1 [24] provides the movie. (b) Time-averaged flow profile for $N_{y}=40$ averaged over four samples $\left(v_{\alpha}=1.0, \sigma=0.3\right)$. The error bars represent the standard deviation. (c) Schematic of the torque forces exerted on the vertices on a boundary $\left(v_{\alpha}>0\right)$. The curved and solid arrows represent the chiral torque generation and the torque forces, respectively.

three regular hexagonal cells as shown in Fig. 1(c), without the line tension fluctuation. When $v_{\alpha}$ is homogeneous, we readily find that the torque force exerted on the vertex $O$ vanishes due to the threefold symmetry. Therefore, even though the torque force is present in the bulk due to cellular deformation, it should be so small that the deformation of cells can readily balance it.

We note that without the line tension fluctuation $(\sigma=0)$, all the cells are just deformed in the asymmetric fashion without continuous cellular flow (see Appendix D). Hence, the cell rearrangements induced by the stochastic fluctuation of the line tension promote the continuous cellular flow.

We next consider a condition in which collective cell migration appears in a defined direction, induced by chiral torque generation. According to a symmetry argument [12], the symmetry along the $y$ axis has to be broken to achieve a unidirectional cellular movement along the $x$ axis. In this paper, we consider a situation where chiral torque generation depends on the position of the cells along the $y$ axis. We assume a linear form given by $v_{\alpha}=-\lambda\left(y_{g}^{\alpha}-L_{y}\right)$, where $y_{g}^{\alpha}$ is the $y$ coordinate of the center of cell $\alpha$. Here, we impose a boundary condition in which the friction coefficients on the top and bottom boundaries are considerably higher $(\eta=10000)$ to avoid the effect of the boundary torque force as we discussed above [Fig. 2(c)]. With the torque gradient, we found that cells migrate unidirectionally in the direction perpendicular to that of the torque gradient [Fig. 3(a)]. A time-averaged flow profile is shown in Fig. 3(b) $\left(\lambda=0.01, \sigma=0.3, N_{y}=40\right)$. With negative $\lambda$, the direction of the cellular migration is reversed. As $\lambda$ increases, the steady-state cellular velocity $V_{\text {ave }}$ in the $x$ axis increases almost linearly [Fig. 3(c)]. These results confirm that the chiral torque gradient is the driving force for this unidirectional collective cell migration. We also found that without the line tension fluctuation, the cells only deform without continuous flow (see Appendix D). Hence, the cell 
(a)

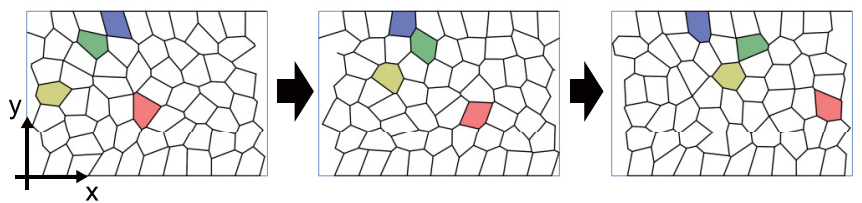

(b)

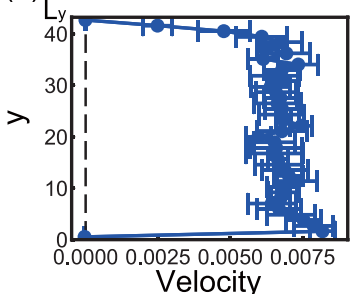

(c)

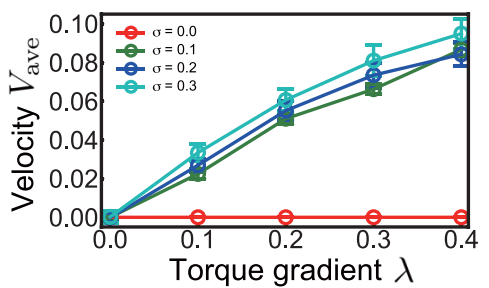

FIG. 3. (a) Time evolution of a cellular configuration in a numerical simulation $\left(\lambda=0.1, \sigma=0.3, N_{y}=6\right)$. The time interval between each configuration is 40 time units. Supplemental Material S2 [24] provides the movie. (b) Time-averaged flow profile for $N_{y}=40$ averaged over five samples $(\lambda=0.01, \sigma=0.3)$. (c) Average velocity $V_{\text {ave }}$ of cells is plotted against torque gradient $\lambda$ for different intensities $\sigma\left(N_{y}=6\right)$. The error bars represent the standard deviation.

rearrangements induced by the stochastic fluctuation in the line tension are necessary for the continuous cellular flow.

In contrast to the case in which the strength of torque is homogeneous, the flow profile shown in Fig. 3(b) indicates that the cells can migrate in the bulk region under the torque gradient. To see the mechanism of the unidirectional cellular migration, we consider the mean-field model of three cells [Fig. 4(a)]. We set the position of the target vertex $O$ as the origin of the coordinate axes, and impose a linear torque gradient $\lambda$ along the $y$ direction. Without loss of generality, we define the center of each surrounding cell as $\overrightarrow{r_{g}^{\alpha}}=$ $l(\cos (2 \pi \alpha / 3+\beta), \sin (2 \pi \alpha / 3+\beta))$, where $l=\sqrt{2 \sqrt{3}} / 3$ is the edge length for the hexagonal regular cell with area $1, \beta$ is an arbitrary constant, and the cell number $\alpha$ is indexed from 1 to 3 . In this configuration, since the force derived from the potential function $E$ vanishes in total owing to the threefold (a)
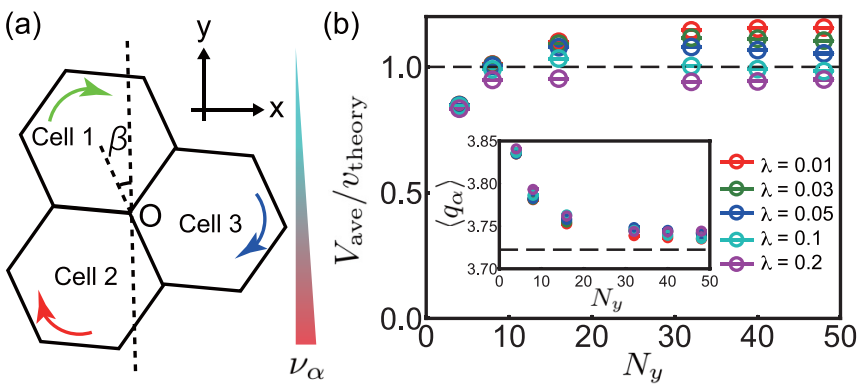

FIG. 4. (a) A cellular configuration for the theoretical analysis on the force exerted on a single vertex $O$. The curved arrows represent the direction of torque generations. (b) The normalized speed $V_{\text {ave }} / v_{\text {theory }}$ is plotted against the number $N_{y}$ of cells for different values of $\lambda$. The dashed line indicates $V_{\text {ave }} / v_{\text {theory }}=1$. (Inset) The dependence of $\left\langle q_{\alpha}\right\rangle$ on $N_{y}$ is shown. The dashed line indicates $\left\langle q_{\alpha}\right\rangle=$ $q_{\text {hex }}$.

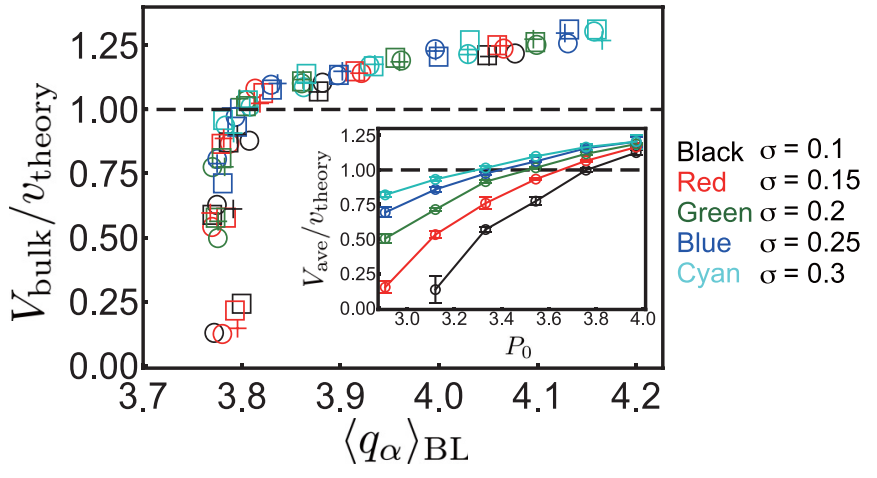

FIG. 5. The normalized bulk velocity $V_{\text {bulk }} / v_{\text {theory }}$ is plotted against the shape index $\left\langle q_{\alpha}\right\rangle_{\mathrm{BL}}$ in the boundary layers for different $\sigma$ and $P_{0}$ for a large system size $N_{y}=40 . \lambda$ is fixed to 0.01 . The different symbols are for different samples. (Inset) The normalized velocity $V_{\text {ave }} / v_{\text {theory }}$ is plotted against the target shape index $P_{0}$ for different values of $\sigma . V_{\text {ave }}$ was averaged over three samples, and the error bars represent the standard deviation.

symmetry, only torque forces from the three cells contribute to the force exerted on the vertex $O$. After a straightforward calculation, we obtain the force $\vec{f}=\left(3 \lambda l^{2} / 2,0\right)$. Consequently, the mean-field model predicts that, when the cells are packed in a regular hexagonal pattern without any boundary, the cells migrate with the speed $v_{\text {theory }}=3 \lambda l^{2} / 2 \eta$.

We tested this prediction numerically using the model without fluctuation in line tension. We apply a small value of $P_{0}=2.90$ to make the cell shape close to regular hexagon. To suppress the boundary effect, we set the friction coefficients of the vertices on both of the bottom and top boundaries equal to those in the bulk, and set the torque force on the vertices on the boundaries to zero. As shown in Fig. 4(b), we found that, by increasing the system size $N_{y}$, the average cell speed $V_{\text {ave }}$ approaches to $v_{\text {theory }}$ for each $\lambda$, confirming that the spatial gradient of torque generated by the cells drives the cellular migration. The deviation from the theoretical value even for the large system size is owing to the inevitable cellular deformation from the regular hexagon assumed in the mean-field model. In the inset of Fig. 4(b), we confirmed the cellular deformation by calculating the average $\left\langle q_{\alpha}\right\rangle$ of the cell shape $q_{\alpha}=P_{\alpha} / \sqrt{A_{\alpha}}$, which equals to $q_{\text {hex }}=2 \sqrt{2} \sqrt[4]{3} \sim 3.72$, when the cells are regular hexagon.

From the above analysis, we found that the cell shape affects the cellular migration velocity. The cell shape can be modulated by altering the target shape index $P_{0}$ or the noise strength $\sigma$. Therefore, we investigated how the migration speed $V_{\text {ave }}$ depends on $P_{0}$ and $\sigma$ (inset of Fig. 5). Here, we set $N_{y}=40, \lambda=0.01$ and applied the same boundary conditions that were used in Fig. 3. We found that an increase of either $P_{0}$ or $\sigma$ enhances the migration speed (inset of Fig. 5). It has been reported that increasing $P_{0}$ lowers the energy barrier for T1 transition to almost zero above a critical value $P_{0}^{*} \sim 3.81$, which is liquid-to-solid transition in confluent tissues [23]. Thus, as $P_{0}$ increases, cell rearrangements occur more frequently. The energy barrier is also overcome by increasing the line tension fluctuation $\sigma$, leading to an increase of the cell rearrangements' frequency. Hence, the migration velocity increases as either $P_{0}$ or $\sigma$ increases (inset of Fig. 5). 
To see the influence of $P_{0}$ and $\sigma$ in a unified way, we focus on the cell shape $q_{\alpha}$ obtained from the numerical simulations. In Fig. 5, we plotted the migration velocity $V_{\text {bulk }}$ in the bulk layers against the cell shape $\left\langle q_{\alpha}\right\rangle_{\mathrm{BL}}$ averaged in the boundary layers, and found that the velocity curves in the inset of Fig. 5 surprisingly collapse onto a single line. Hence, $\left\langle q_{\alpha}\right\rangle_{\mathrm{BL}}$ is an excellent indicator of the migration velocity in our model. Here, we define the boundary and bulk layers from the flow profile, and exclude the cells on the bottom and top boundaries for calculation of $\left\langle q_{\alpha}\right\rangle_{\mathrm{BL}}$ since the cells are strongly deformed by the flat boundaries (see Appendix E).

We discuss how the bulk velocity $V_{\text {bulk }}$ is uniquely determined by the cell shape $\left\langle q_{\alpha}\right\rangle_{\mathrm{BL}}$ in Fig. 5. The cellular velocity should be determined by the balance between the bulk torque force and how easily cells rearrange over the energy barrier of $\mathrm{T} 1$ transition in the boundary layers. In the present model, since the torque force depends on the cell shape, the bulk torque force should be determined by the cell shape $\left\langle q_{\alpha}\right\rangle_{\mathrm{BU}}$ averaged in the bulk. The cell shape $q_{\alpha}$ has been also suggested as an indicator of how easily cells rearrange over the energy barrier of $\mathrm{T} 1$ transition $[25,26]$. Hence, the energy barrier of $\mathrm{T} 1$ transition in the boundary layers depends on $\left\langle q_{\alpha}\right\rangle_{\mathrm{BL}}$. Consequently, considering that $\left\langle q_{\alpha}\right\rangle_{\mathrm{BL}} \approx\left\langle q_{\alpha}\right\rangle_{\mathrm{BU}}$ is satisfied (see Appendix F), and hence both bulk torque force and energy barrier of T1 transition depend on $\left\langle q_{\alpha}\right\rangle_{\mathrm{BL}},\left\langle q_{\alpha}\right\rangle_{\mathrm{BL}}$ uniquely determines the migration velocity.

\section{CONCLUSION}

In this paper, we have reported that a dynamical chiral property at the single-cell level, which is cellular chiral torque generation, can induce collective cellular migration. Our model predicts that, when the strength of torque is spatially homogeneous, the torque forces in the bulk almost balance, and those generated by the cells at the boundaries drive the bidirectional cellular migration. Another prediction is that, under the gradient of chiral torque strength, the cells can migrate unidirectionally and perpendicularly to the gradient driven by the torque force generated in the bulk. Although the mechanism of the torque generation at the single-cell level is not fully revealed experimentally, previous studies reported that a combination of cytoskeleton and motor proteins generates the cellular torque. Activity of these proteins is regulated by various biochemical pathways such as Rho signaling pathway, which can be further regulated by extracellular signals $[27,28]$. Hence, the spatial gradient of cellular torque strength can be produced by a gradient of such an extracellular signal. In in vivo systems, an epithelial tissue is attached to other tissues, which may emit such extracellular signals that form concentration gradients. Since both the cellular chiral torque generation and the gradient of the cellular torque can exist in in vivo situation, we expect that the mechanism of the LR symmetry breaking in tissue dynamics we have proposed plays an essential role in chiral collective cellular movements, such as the twist of epithelial tube [7] and the unidirectional epithelial cellular flow [9] during development. Remarkably, in a recent experimental study, Ray et al. reported that chick cardiac cells extracted from embryonic heart exhibit chiral rotational motion, and such a single-cellular chirality correlates with the handedness of the loop structure of the chick heart [29]. Our theoretical prediction will promote experimental studies in this line by proposing that the spatial gradient of cellular chirality can induce the tissue level dynamics which can cause the left-right asymmetric structure formation.

\section{ACKNOWLEDGMENTS}

We thank M. Tarama and S. Tarama for critical reading of the manuscript. This work is supported by Grant-in-Aid for JSPS Fellows (Grant No. 18J01239 to T.Y.), KAKENHI Grants No. 17H07366 (to T.Y.), No. JP16K17777 (to T.H.), No. JP19K03764 (to T.H.), and No. JP19H00996 (to T.S.), Research Grant from HFSP (Ref. Grant No. RGY0081/2019) (to T.Y.), the Inamori Foundation (to T.H.), and JST CREST Grant No. JPMJCR1852, Japan (to T.S.).

\section{APPENDIX A: DEFINITION OF THE AREA CENTROID OF A CELL}

We define the cell center of cell $\alpha$ using the area centroid as $\vec{r}_{g}^{\alpha}=\frac{1}{6 A_{\alpha}} \sum_{\mu=1}^{N_{\alpha}}\left(\vec{r}_{\mu}^{\alpha}+\vec{r}_{\mu+1}^{\alpha}\right)\left(\vec{r}_{\mu}^{\alpha} \times \vec{r}_{\mu+1}^{\alpha}\right) \cdot \vec{n}$. Here, $\mu$ is the index of the $N_{\alpha}$ vertices around the cell $\alpha$, indexed in the counterclockwise direction. The position vector of each vertex is represented by $\vec{r}_{\mu}^{\alpha}$ and $\vec{r}_{N_{\alpha}+1}^{\alpha}=\vec{r}_{1}^{\alpha}$.

\section{APPENDIX B: DISCUSSION ON THE TORQUE FORCE FROM THE THREE-DIMENSIONAL PERSPECTIVE}

We here justify the torque force introduced by Eq. (4) from the three-dimensional (3D) perspective. It has been reported for the epithelial tissues moving on the rigid basal matrix that the apical side is indeed forming polygonal meshwork thanks to the apical actomyosin belt but the basolateral side can independently move with the protrusive activities [30,31]. The cell-cell adhesion molecule is mostly localized along the adherens junction at the apical side and the lateral side below the adherens junction is considered to be mechanically independent between neighboring cells [Fig. 1(b)]. Based on this fact, in what follows, we propose a simple 3D model which takes into account both the apical and basal sides and elaborate how we can derive our model, 2D vertex model with torque force $\vec{T}_{i}^{\alpha}$ given by Eq. (4).

In our model, we represent each cell (cell $\alpha$ ) in the tissue as two rotating rigid disks at apical and basal sides connected through the rigid shaft directing almost perpendicular to the substrate (this direction is represented by a unit normal vector $\vec{n}$ from the basal to apical sides in the following). We assume the $z$ coordinates of the apical and basal sides, i.e., the length of the shaft, are constant by neglecting the change in height of the cells. Dynamics of apical and basal disks are assumed to be driven by a torque dipole consisting of two torque monopoles $\vec{\tau}_{m}^{\alpha}=\tau_{m}^{\alpha} \vec{n}$ and $-\vec{\tau}_{m}^{\alpha}$, respectively, as schematically shown in Fig. 1(a). We define the angular velocity of the apical and basal sides as $\vec{\omega}_{a}^{\alpha}=\omega_{a}^{\alpha} \vec{n}$ and $\vec{\omega}_{b}^{\alpha}=\omega_{b}^{\alpha} \vec{n}$, respectively. We neglect the twist elasticity of the cell since the continuously rotating apical surface, which has been observed experimentally in $[15,16]$, is realized only when the twist viscosity is dominant over the twist elasticity. We represent the $x y$ 
coordinates of the centers of the apical and basal planes of the target cell $\alpha$ as $\vec{R}_{a}^{\alpha}$ and $\vec{R}_{b}^{\alpha}$, respectively.

We start with the force and torque balance equations at the apical side of each cell

$$
\begin{gathered}
\zeta_{c}^{\alpha}\left(\dot{\vec{R}}_{a}^{\alpha}-\dot{\vec{R}}_{b}^{\alpha}\right)=\vec{h}^{\alpha}\left(\vec{R}_{a}^{\alpha}-\vec{R}_{b}^{\alpha}\right)+\vec{f}_{e}^{\alpha}, \\
\gamma_{c}^{\alpha}\left(\omega_{a}^{\alpha}-\omega_{b}^{\alpha}\right)=\tau_{m}^{\alpha}+\tau_{\mathrm{e}}^{\alpha},
\end{gathered}
$$

respectively, and those at the basal side

$$
\begin{gathered}
\xi_{s}^{\alpha} \dot{\vec{R}}_{b}^{\alpha}+\zeta_{c}^{\alpha}\left(\dot{\vec{R}}_{b}^{\alpha}-\dot{\vec{R}}_{a}^{\alpha}\right)=-\vec{h}^{\alpha}\left(\vec{R}_{a}^{\alpha}-\vec{R}_{b}^{\alpha}\right), \\
\eta_{s}^{\alpha} \omega_{b}^{\alpha}+\gamma_{c}^{\alpha}\left(\omega_{b}^{\alpha}-\omega_{a}^{\alpha}\right)=-\tau_{m}^{\alpha} .
\end{gathered}
$$

Here, the vectors in Eqs. (B1) and (B3) are two-dimensional vectors in the $x y$ planes in either the apical or the basal sides. The coefficients $\gamma_{c}^{\alpha}$ and $\eta_{s}^{\alpha}$ are the rotational viscous coefficient of the cell and the rotational friction coefficient between the cell and the substrate, respectively. The function $\vec{h}^{\alpha}$ with respect to $\left(\vec{R}_{a}^{\alpha}-\vec{R}_{b}^{\alpha}\right)$ represents the intracellular elastic forces. The force $\vec{f}_{e}^{\alpha}$ and the torque $\vec{\tau}_{e}^{\alpha}=\tau_{e}^{\alpha} \vec{n}$ are the contribution from interactions with neighboring cells. In general, the torque generated by the neighboring cells contributes to both $\vec{f}_{e}^{\alpha}$ and $\vec{\tau}_{e}^{\alpha}$ via the intercellular interactions. $\zeta_{c}^{\alpha}$ is the viscous coefficient of the intracellular dissipative forces, and $\xi_{s} \dot{\vec{R}}_{b}^{\alpha}$ is the friction force between the cell and the substrate. The terms $\xi_{s}^{\alpha} \dot{\vec{R}}_{b}^{\alpha}$ and $\eta_{s}^{\alpha} \omega_{b}^{\alpha}$ explicitly take into account the interaction between the cell and the substrate at the basal side.

From the force balance equations (B1) and (B3), we obtain $\xi_{s} \dot{\vec{R}}_{b}=\vec{f}_{e}^{\alpha}$. We here assume that the differential elastic modulus $-\partial \vec{h}^{\alpha}(\vec{x}) /\left.\partial \vec{x}\right|_{\vec{x}=0}$ is large enough so that the centers of the apical and basal planes should be almost identical, i.e., $\vec{R}_{a}^{\alpha} \sim \vec{R}_{b}^{\alpha}$. In this limit, we can approximately describe the translational dynamics of the apical side by

$$
\xi_{s}^{\alpha} \dot{\vec{R}}_{a}^{\alpha}=\vec{f}_{e}^{\alpha} .
$$

Furthermore, by solving the torque balance equations (B2) and (B4), we obtain

$$
\begin{gathered}
\frac{\eta_{s}^{\alpha} \gamma_{c}^{\alpha}}{\eta_{s}^{\alpha}+\gamma^{\alpha}} \omega_{a}^{\alpha}=\frac{\eta_{s}^{\alpha}}{\eta_{s}^{\alpha}+\gamma^{\alpha}} \tau_{m}^{\alpha}+\tau_{e}^{\alpha}, \\
\eta_{s}^{\alpha} \omega_{b}^{\alpha}=\tau_{e}^{\alpha} .
\end{gathered}
$$

These expressions suggest that the rotational dynamics of the apical and basal sides can be independently described by the chiral torque $\tau_{m}^{\alpha}$ and the torque $\tau_{e}^{\alpha}$ exerted via the intercellular interaction at the apical side.

At this point, no assumptions have been made for the interactions between cells. Therefore, we cannot determine $\vec{f}_{e}^{\alpha}$ and $\tau_{e}^{\alpha}$, and hence the governing equation for the overall tissue dynamics is not obtained. In what follows, therefore, we will take into account the interaction between cells and investigate how torque force generation affects the apical 2D vertex model. For this purpose, we will provide the case studies of this model, in the divided subsections. The first subsection is devoted to the simplest model in which each apical disk interacts with their neighbor apical disks as perfectly engaging gears to get the first intuition. The second subsection is devoted to an alternative case from which we can derive the $2 \mathrm{D}$ vertex model with torque force.

\section{The simplest model: Cells in a tissue as perfectly engaging gears with torque generation}

We first study the simplest model in which each apical disk interacts with their neighbor apical disks as perfectly engaging gears. Due to the torque dipole generated by the neighboring cells, each cell (cell $\alpha$ ) experiences the force $\vec{f}_{e}^{\alpha}$ and the torque $\vec{\tau}_{e}^{\alpha}=\tau_{e}^{\alpha} \vec{n}$ from the neighboring cells. One may wonder how the torque $\vec{\tau}_{m}^{\beta}$ generated in each neighboring cell (cell $\beta$ ) can contribute to $\vec{f}_{e}^{\alpha}$ and the torque $\vec{\tau}_{e}^{\alpha}$, which is important later to provide the effect of the torque dipole in the tissue case. We here roughly estimate it assuming that the apical disks do not slide with each other. Assuming that each of the cells surrounding the cell $\beta$ is subjected to the force $\vec{f}^{w, \beta}$ from the cell $\beta\left(w=1, \ldots, N_{\beta \mathrm{NC}}\right.$ with the number $N_{\beta \mathrm{NC}}$ of the neighbors of the cell $\beta$ ), its reaction force $-\vec{f}^{w, \beta}$ is the origin of $\vec{\tau}_{e}^{\beta}$. Since we have multiple cells around each cell, we estimate as $\vec{\tau}_{e}^{\beta} \sim \sum_{w \in \beta \mathrm{NC}} \rho^{\beta} \vec{c}_{w, \beta} \times\left(-\vec{f}^{w, \beta}\right)$. Here, $\rho^{\beta}$ is the radius of the apical disk, $\vec{c}_{w, \beta}$ is the unit vector directing from centers of cell $\beta$ to cell $w$, and $\times$ represents the cross product. The summation $\sum_{w \in \beta \mathrm{NC}}$ runs over all the neighbor cells of the cell $\beta$.

We next define the torque $\vec{\tau}_{m}^{\prime \beta}=\tau_{m}^{\prime \beta} \vec{n}$ as the torque which cell $\beta$ exerts on the surrounding cells in total. The torque balance condition on cell $\beta$ gives $\vec{\tau}_{m}^{\prime \beta}+\vec{\tau}_{e}^{\beta}=0$, and hence $\vec{\tau}_{m}^{\prime \beta} \sim$ $\sum_{w \in \beta \mathrm{NC}} \rho^{\beta} \vec{c}_{w, \beta} \times \vec{f}^{w, \beta}$. By assuming that cells are packed almost in the regular hexagonal pattern, we approximately have $\vec{f}^{w, \beta} \sim{\overrightarrow{\tau_{m}^{\prime}}}^{\beta} \times \vec{c}_{w, \beta} /\left(\rho^{\beta} N_{\beta \mathrm{NC}}\right)$. Therefore, we obtain

$$
\vec{\tau}_{e}^{\alpha} \sim \rho^{\alpha} \sum_{\beta \in \alpha \mathrm{NC}} \vec{c}_{\beta, \alpha} \times \vec{f}^{\alpha, \beta}=-\rho^{\alpha} \sum_{\beta \in \alpha \mathrm{NC}} \vec{\tau}_{m}^{\prime \beta} /\left(\rho^{\beta} N_{\beta \mathrm{NC}}\right)
$$

and

$$
\vec{f}_{e}^{\alpha} \sim \sum_{\beta \in \alpha \mathrm{NC}} \vec{f}^{\alpha, \beta}=\sum_{\beta \in \alpha \mathrm{NC}}\left({\overrightarrow{\tau_{m}^{\prime}}}^{\beta} \times \vec{c}_{\alpha, \beta}\right) /\left(\rho^{\beta} N_{\beta \mathrm{NC}}\right) .
$$

Finally, since $\omega_{b}^{\beta}=\left(-\tau_{m}^{\beta}+\gamma_{c}^{\beta} \omega_{a}^{\beta}\right) /\left(\eta_{s}^{\beta}+\gamma_{c}^{\beta}\right)$ from Eqs. (B6) and (B7), the net torque can be writtten as $\tau_{m}^{\prime \beta}=-\tau_{e}^{\beta}=\frac{\eta_{s}^{\beta}}{\eta_{s}^{\beta}+\gamma_{c}^{\beta}}\left(\tau_{m}^{\beta}-\gamma_{c}^{\beta} \omega_{a}^{\beta}\right)$. Therefore, from Eq. (B8), the external torque is given by

$$
\begin{aligned}
\tau_{\mathrm{e}}^{\alpha}= & -\sum_{\beta \in \alpha \mathrm{NC}} \frac{1}{N_{\beta \mathrm{NC}}} \frac{\rho^{\alpha}}{\rho^{\beta}} \frac{\eta_{s}^{\beta}}{\eta_{s}^{\beta}+\gamma_{c}^{\beta}} \tau_{m}^{\beta} \\
& +\sum_{\beta \in \alpha \mathrm{NC}} \frac{1}{N_{\beta \mathrm{NC}}} \frac{\rho^{\alpha}}{\rho^{\beta}} \frac{\eta_{s}^{\beta} \gamma_{c}^{\beta}}{\eta_{s}^{\beta}+\gamma_{c}^{\beta}} \omega_{a}^{\beta} .
\end{aligned}
$$

The first term on the right-hand side, which is originated from the torque dipole of the neighboring cells, drives the rotation of cell $\alpha$. The second term provides a dissipation through the neighboring cells around cell $\alpha$. The cell's rotational dynamics eventually follows Eq. (B6) with Eq. (B10), which are 
together rewritten as

$$
\begin{aligned}
& \sum_{\beta \in \alpha \mathrm{NC}}\left(\frac{1}{N_{\alpha \mathrm{NC}}} \frac{1}{\rho^{\alpha}} \frac{\eta_{s}^{\alpha} \gamma_{c}^{\alpha}}{\eta_{s}^{\alpha}+\gamma^{\alpha}} \omega_{a}^{\alpha}-\frac{1}{N_{\beta \mathrm{NC}}} \frac{1}{\rho^{\beta}} \frac{\eta_{s}^{\beta} \gamma_{c}^{\beta}}{\eta_{s}^{\beta}+\gamma_{c}^{\beta}} \omega_{a}^{\beta}\right) \\
& =\sum_{\beta \in \alpha \mathrm{NC}}\left(\frac{1}{N_{\alpha \mathrm{NC}}} \frac{1}{\rho^{\alpha}} \frac{\eta_{s}^{\alpha}}{\eta_{s}^{\alpha}+\gamma^{\alpha}} \tau_{m}^{\alpha}-\frac{1}{N_{\beta \mathrm{NC}}} \frac{1}{\rho^{\beta}} \frac{\eta_{s}^{\beta}}{\eta_{s}^{\beta}+\gamma_{c}^{\beta}} \tau_{m}^{\beta}\right) .
\end{aligned}
$$

This equation of rotational motion suggests the nonlocal nature such that the friction dominating the angular velocity $\omega_{a}$ of each cell is determined by the dissipation parameters of not only the cell itself but also the other cells $\gamma_{c}^{\beta}$ and $\eta_{s}^{\beta}$. Similarly, the external force is given by

$$
\begin{aligned}
\vec{f}_{e}^{\alpha} \sim & \sum_{\beta \in \alpha \mathrm{NC}} \frac{1}{N_{\beta \mathrm{NC}}} \frac{1}{\rho^{\beta}} \frac{\eta_{s}^{\beta}}{\eta_{s}^{\beta}+\gamma_{c}^{\beta}}\left[\vec{c}_{\alpha, \beta} \times\left(-\vec{\tau}_{m}{ }^{\beta}\right)\right] \\
& +\sum_{\beta \in \alpha \mathrm{NC}} \frac{1}{N_{\beta \mathrm{NC}}} \frac{1}{\rho^{\beta}} \frac{\eta_{s}^{\beta} \gamma_{c}^{\beta}}{\eta_{s}^{\beta}+\gamma_{c}^{\beta}}\left(\vec{c}_{\alpha, \beta} \times \vec{\omega}_{a}^{\beta}\right)
\end{aligned}
$$

with $\vec{\omega}_{a}{ }^{\beta}=\omega_{a}^{\beta} \vec{n}$. The first term explains the origin of the functional form of the torque force given by Eq. (4). The second term is subtracting part of the torque in the neighboring cells which is balanced with the viscosity for torsional dynamics inside the cell. The cell's translational dynamics eventually follows Eq. (B5) with Eq. (B12). Therefore, the external force $\vec{f}_{e}^{\alpha}$ consists of the contribution from the torque generated in the neighboring cells, i.e., torque force $\vec{T}_{i}$, and the friction at the neighbors. The neighbors' rotation $\vec{\omega}_{a}{ }^{\beta}$ is given through Eq. (B11), so that the each cell's dynamics is determined fully globally.

\section{Model for cells with torque generation in an epithelial monolayer on a rigid substrate}

In this section, we demonstrate a case study with an example model to derive Eq. (4). In addition to the disk-shaft model given by Eqs. (1)-(4) for cells' inner bodies, we here introduce a $2 \mathrm{D}$ vertex model as a frame of the apical side, which describes the apical actomyosin belt and cortex, satisfying the force balance

$$
-\frac{\partial E\left(\left\{\vec{r}_{i}\right\}\right)}{\partial \vec{r}_{i}}+\vec{F}_{\mathrm{fc} i}=0 .
$$

Here, $\vec{F}_{\mathrm{fc}_{i}}=\vec{F}_{\mathrm{fc}_{i}}^{\alpha}+\vec{F}_{\mathrm{fc}_{i}}^{\beta}+\vec{F}_{\mathrm{fc}_{i}}^{\gamma}$ represents the force from the inner bodies of the three cells $(\alpha, \beta, \gamma)$ surrounding the $i$ th vertex, and $\vec{F}_{\mathrm{fc}_{i}}^{\alpha}$ is the contribution specifically from cell $\alpha$. $E\left(\left\{\vec{r}_{i}\right\}\right)$ is the potential of the conventional 2D vertex model. We here assume that the inner dynamics of each cell [Eqs. (B1)-(B4)] interacts only with the vertices of the cell.

Since the inner body of cell $\alpha$ exerts the force $\vec{F}_{\mathrm{fc}_{i} i}^{\alpha}$ on the $i$ th vertex, the reaction force $-\vec{F}_{\mathrm{fc}_{i}}^{\alpha}$ is exerted on the inner body. Furthermore, since each cell is subjected to the force $\vec{f}_{e}^{\alpha}$ and torque $\vec{\tau}_{\mathrm{e}}^{\alpha}=\tau_{\mathrm{e}}^{\alpha} \vec{n}$ from the neighboring cells via the frame of the $2 \mathrm{D}$ vertex model, $\vec{f}_{e}^{\alpha}$ and $\vec{\tau}_{\mathrm{e}}^{\alpha}$ are related with $\vec{F}_{\mathrm{fc} i}$ through

$$
\vec{f}_{e}^{\alpha}=\sum_{i \in \alpha}\left(-\vec{F}_{\mathrm{fc}_{i}}^{\alpha}\right)
$$

and

$$
\tau_{\mathrm{e}}^{\alpha} \vec{n}=\sum_{i \in \alpha}\left(\vec{r}_{i}-\vec{r}_{g}^{\alpha}\right) \times\left(-\vec{F}_{\mathrm{fc}_{i}}^{\alpha}\right),
$$

where $\sum_{i \in \alpha}$ runs over all the vertices $i$ of cell $\alpha$.

Let us investigate the possible functional form of the force $\vec{F}_{\mathrm{fc}_{i}}^{\alpha}$. We assume that there is no sliding between the cells. In this section, this assumption is realized by matching the dynamics of the vertex model frame with the cell inner dynamics as

$$
\dot{\vec{R}}_{a}^{\alpha}=\frac{1}{N_{\alpha}} \sum_{i \in \alpha} \frac{d}{d t} \vec{r}_{i}
$$

and

$$
\omega_{a}^{\alpha} \vec{n}=\frac{1}{N_{\alpha}} \sum_{i \in \alpha}\left(\vec{r}_{i}-\vec{r}_{g}^{\alpha}\right) \times \frac{d}{d t}\left(\vec{r}_{i}-\vec{r}_{g}^{\alpha}\right)
$$

with the number of vertices $N_{\alpha}$ on cell $\alpha$. The force $\vec{F}_{\mathrm{fc}_{i}}^{\alpha}$ is determined to satisfy these conditions. For the convenience to discuss this, we split it into two terms as $\vec{F}_{\mathrm{fc}_{i}}^{\alpha}=\vec{F}_{\mathrm{fc}_{i}}^{\alpha, \tau}+\vec{F}_{\mathrm{fc}_{i}}^{\alpha, f}$, where $\vec{F}_{\mathrm{fc}_{i}}^{\alpha, f}$ is torque-free part, satisfying $\sum_{i \in \alpha}\left(\vec{r}_{i}-\vec{r}_{g}^{\alpha}\right) \times \vec{F}_{\mathrm{fc}_{i}}^{\alpha, f}=0$, while $\vec{F}_{\mathrm{fc}_{i}}^{\alpha, \tau}$ is the contribution to the torque. Let us focus on the torque part in the following, and find out the functional form of $\vec{F}_{\mathrm{fc} i}{ }^{\alpha, \tau}$ satisfying the non-slip condition [Eq. (B17)].

We have obtained $\omega_{b}^{\beta}=\left(-\tau_{m}^{\beta}+\gamma_{c}^{\beta} \omega_{a}^{\beta}\right) /\left(\eta_{s}^{\beta}+\gamma_{c}^{\beta}\right)$ from Eq. (B4). Using this expression with Eqs. (B2) and (B15), we obtain the relation

$$
\frac{\gamma_{c}^{\alpha} \eta_{s}^{\alpha}}{\eta_{s}^{\alpha}+\gamma_{c}^{\alpha}} \omega_{a}^{\alpha} \vec{n}+\frac{\eta_{s}^{\alpha}}{\eta_{s}^{\alpha}+\gamma_{c}^{\alpha}} \tau_{m}^{\alpha} \vec{n}=-\sum_{i \in \alpha}\left(\vec{r}_{i}-\vec{r}_{g}^{\alpha}\right) \times \vec{F}_{\mathrm{fc}_{i}}^{\alpha, \tau} .
$$

Equation (B18) contains $2 N_{\alpha}$ degrees of freedom for $\vec{F}_{\mathrm{fc}_{i}}{ }^{\alpha, \tau}$ for $i \in \alpha$, while the equation itself is only a single scalar constraint. Therefore, it is in principle impossible to determine a unique functional form of $\vec{F}_{\mathrm{fc}_{i}}{ }^{\alpha, \tau}$ from this simple model. We will here find one of the possible functional forms. For this, we put an ansatz

$$
\vec{F}_{\mathrm{fc}_{i}}^{\alpha, \tau}=\tilde{v}_{\alpha}\left(\vec{r}_{i}-\vec{r}_{g}^{\alpha}\right) \times \vec{n} .
$$

We investigate whether this functional form can satisfy the the non-slip condition given by Eq. (B17) with Eq. (B18). Importantly, due to the formula for cross product $\left(\vec{r}_{i}-\vec{r}_{g}^{\alpha}\right) \times\left[\left(\vec{r}_{i}-\right.\right.$ $\left.\left.\vec{r}_{g}^{\alpha}\right) \times \vec{n}\right]=-\left|\vec{r}_{i}-\vec{r}_{g}^{\alpha}\right|^{2} \vec{n}$, the right-hand side of Eq. (B18) can

(a)

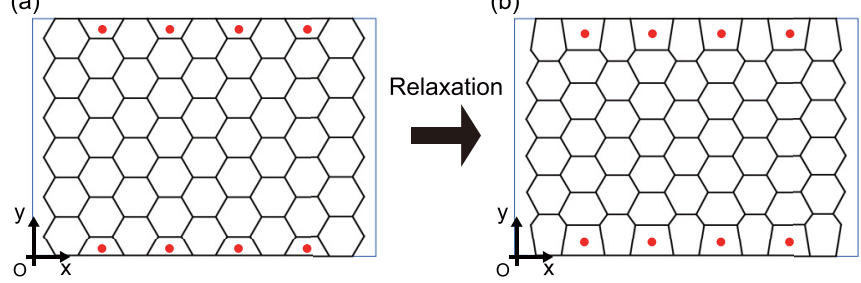

FIG. 6. (a) The initial condition with regular hexagonal cells. The half regular hexagonal cells on the boundaries are shown with red dots. (b) A configuration after relaxation with $\sigma=0$ and $v_{\alpha}=0$ $\left(P_{0}=3.54\right)$. 
have the same directionality with the left-hand side $(\vec{n})$ when $\vec{F}_{\mathrm{fc}_{i}}^{\alpha, \tau} \|\left(\vec{r}_{i}-\vec{r}_{g}^{\alpha}\right) \times \vec{n}$ as given in Eq. (B19). Therefore, substituting Eq. (B17) into Eq. (B18) gives $\frac{\gamma_{c}^{\alpha} \eta_{s}^{\alpha}}{\eta_{s}^{\alpha}+\gamma_{c}^{\alpha}} \omega_{a}^{\alpha}+\frac{\eta_{s}^{\alpha}}{\eta_{s}^{\alpha}+\gamma_{c}^{\alpha}} \tau_{m}^{\alpha}=$ $\tilde{v}_{\alpha} \sum_{i \in \alpha}\left|\vec{r}_{i}-\vec{r}_{g}^{\alpha}\right|^{2}$ with $\omega_{a}^{\alpha}$ given in Eq. (B17). This means that the ansatz [Eq. (B19)] is valid and the unknown coefficient has to be

$$
\tilde{v}_{\alpha}=\frac{1}{\sum_{i \in \alpha}\left|\vec{r}_{i}-\vec{r}_{g}^{\alpha}\right|^{2}}\left(\frac{\gamma_{c}^{\alpha} \eta_{s}^{\alpha}}{\eta_{s}^{\alpha}+\gamma_{c}^{\alpha}} \frac{1}{N_{\alpha}} \sum_{i \in \alpha} \vec{n} \cdot\left[\left(\vec{r}_{i}-\vec{r}_{g}^{\alpha}\right) \times \frac{d}{d t}\left(\vec{r}_{i}-\vec{r}_{g}^{\alpha}\right)\right]+\frac{\eta_{s}^{\alpha}}{\eta_{s}^{\alpha}+\gamma_{c}^{\alpha}} \tau_{m}^{\alpha}\right) .
$$

The translational part $\vec{F}_{\mathrm{fc}_{i}}^{f} \equiv{\overrightarrow{F_{\mathrm{fc}}}}_{i}^{\alpha, f}+{\overrightarrow{F_{\mathrm{fc}}}}_{i}^{\beta, f}+{\overrightarrow{F_{\mathrm{fc}}}}_{i}^{\gamma, f}$ can be calculated based on Eq. (B16) in a similar manner. By substituting Eqs. (B16) and (B14) into the apical approximation (B5), and then using the allocation $\vec{F}_{\mathrm{fc}_{i}}^{\alpha}=\vec{F}_{\mathrm{fc}_{i}}^{\alpha, \tau}+\vec{F}_{\mathrm{fc}_{i}}^{\alpha, f}$ and the torque force assumed in Eq. (B19), we have $\sum_{i \in \alpha} \vec{F}_{\mathrm{fc}_{i}}^{\alpha, f}=-\xi_{s}^{\alpha} \sum_{i \in \alpha} \frac{d}{d t} \vec{r}_{i}-\frac{\tilde{\nu}_{\alpha}}{N_{\alpha}} \sum_{i \in \alpha}\left(\vec{r}_{i}-\vec{r}_{g}^{\alpha}\right) \times \vec{n}$ with $\tilde{v}_{\alpha}$ which has been given by Eq. (B20). Here, again by evenly distributing the force exerted on the cell among the vertices, i.e., $\vec{F}_{\mathrm{fc}_{i}}^{\alpha, f}=\frac{1}{N_{\alpha}} \sum_{i \in \alpha} \vec{F}_{\mathrm{fc}_{i}}^{\alpha, f}$, the translational part is obtained as

$$
\vec{F}_{\mathrm{fc}_{i}}^{\alpha, f}=-\frac{\xi_{s}^{\alpha}}{N_{\alpha}^{2}} \sum_{j \in \alpha} \frac{d}{d t} \vec{r}_{j}-\frac{\tilde{v}_{\alpha}}{N_{\alpha}} \sum_{j \in \alpha}\left(\vec{r}_{j}-\vec{r}_{g}^{\alpha}\right) \times \vec{n} .
$$

Finally, substituting Eqs. (B19), (B20), and (B21) into (B13) gives the 2D cellular vertex model

$$
\vec{\Xi}_{i}\left(\left\{\vec{r}_{i}\right\}\right)=-\frac{\partial E\left(\left\{\vec{r}_{i}\right\}\right)}{\partial \vec{r}_{i}}+\vec{T}_{i}
$$

where the nonlinear nonlocal friction resistance $\vec{\Xi}_{i}=\vec{\Xi}_{i}^{\alpha}+\vec{\Xi}_{i}^{\beta}+\vec{\Xi}_{i}^{\gamma}$ is given by

$$
\begin{aligned}
\vec{\Xi}_{i}^{\alpha}\left(\left\{\vec{r}_{i}\right\}\right)= & \frac{\xi_{s}^{\alpha}}{N_{\alpha}^{2}} \sum_{j \in \alpha} \frac{d}{d t} \vec{r}_{j}-\frac{\gamma_{c}^{\alpha} \eta_{s}^{\alpha}}{\eta_{s}^{\alpha}+\gamma_{c}^{\alpha}} \frac{1}{\sum_{j \in \alpha}\left|\vec{r}_{j}-\vec{r}_{g}^{\alpha}\right|^{2}} \frac{1}{N_{\alpha}} \sum_{j \in \alpha} \vec{n} \cdot\left[\left(\vec{r}_{j}-\vec{r}_{g}^{\alpha}\right) \times \frac{d}{d t}\left(\vec{r}_{j}-\vec{r}_{g}^{\alpha}\right)\right] \\
& +\frac{\gamma_{c}^{\alpha} \eta_{s}^{\alpha}}{\eta_{s}^{\alpha}+\gamma_{c}^{\alpha}} \frac{1}{\sum_{j \in \alpha}\left|\vec{r}_{j}-\vec{r}_{g}^{\alpha}\right|^{2}} \frac{1}{N_{\alpha}} \sum_{j \in \alpha} \vec{n} \cdot\left[\left(\vec{r}_{j}-\vec{r}_{g}^{\alpha}\right) \times \frac{d}{d t}\left(\vec{r}_{j}-\vec{r}_{g}^{\alpha}\right)\right]\left(\frac{1}{N_{\alpha}} \sum_{j \in \alpha}\left(\vec{r}_{j}-\vec{r}_{g}^{\alpha}\right) \times \vec{n}\right)
\end{aligned}
$$

and the torque force $\vec{T}_{i}=\vec{T}_{i}^{\alpha}+\vec{T}_{i}^{\beta}+\vec{T}_{i}^{\gamma}$ is given by

$$
\vec{T}_{i}^{\alpha}=\frac{\eta_{s}^{\alpha}}{\eta_{s}^{\alpha}+\gamma_{c}^{\alpha}} \frac{\tau_{m}^{\alpha}}{\sum_{j \in \alpha}\left|\vec{r}_{j}-\vec{r}_{g}^{\alpha}\right|^{2}}\left[\left(\vec{r}_{i}-\vec{r}_{g}^{\alpha}\right) \times \vec{n}-\frac{1}{N_{\alpha}} \sum_{j \in \alpha}\left(\vec{r}_{j}-\vec{r}_{g}^{\alpha}\right) \times \vec{n}\right] .
$$

In this way, we obtain the $2 \mathrm{D}$ cellular vertex model used in the main text, but here we also have complicated nonlinear friction terms [Eq. (B23)] and the drift-correction term [the second term in the square brackets on the right hand side of Eq. (B24)]. The first term of Eq. (B24) introduces the "torque force" into the $2 \mathrm{D}$ cellular vertex model. Remember that, although this is not the unique possible functional form of the torque force $\vec{T}_{i}^{\alpha}$, this solid analysis sufficiently proved that Eq. (B19) satisfies all the mechanical requirements, namely, action-reaction laws and force balances at the apical and basal sides. This is indeed the 2D cellular vertex model and torque force with the functional form assumed in Eq. (4), with the following simplifications: (i) We decided to neglect the nonlinearity and nonlocality of the friction term and simplify the friction as the linear friction with a scalar coefficient because studying the influence of the exact form of the friction is a subject far beyond the scope of this paper. (ii) We neglected the complicated dependence of the coefficients of the torque force on the cell size $\sum_{i \in \alpha}\left|\vec{r}_{i}-\vec{r}_{g}^{\alpha}\right|^{2}$ for the same reason. (iii) We neglected the drift-correction term [the second term in the square brackets on the right-hand side of Eq. (B24)] because this term becomes negligible when the tissue morphology is close to the regular hexagonal packing. These simplifications may not cause serious influences on the translational motion of cells.

In conclusion, through the derivation of the $2 \mathrm{D}$ cellular vertex model based on an example, the consistency of our model with Eq. (4) with the mechanical requirements was confirmed [Eq. (B22)]. Furthermore, we have clarified what kind of (a)

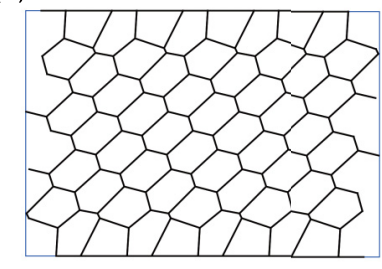

(b)

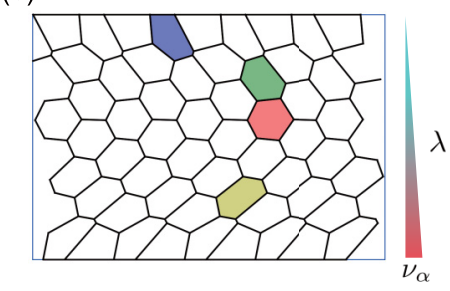

FIG. 7. The tissue deformation observed without continuous cellular flow in the zero-noise limit $\sigma=0$ (a) under a homogeneous cellular chiral torque $\left(v_{\alpha}=0.2\right)$ and (b) under a gradient of cellular chiral torque $(\lambda=0.1)$. 
(a)

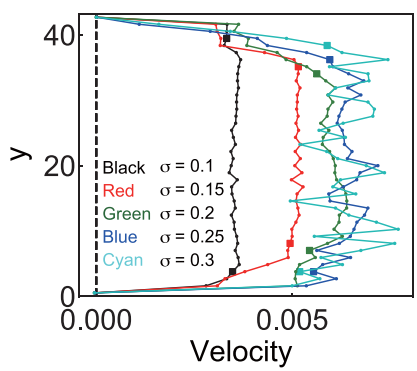

(b)

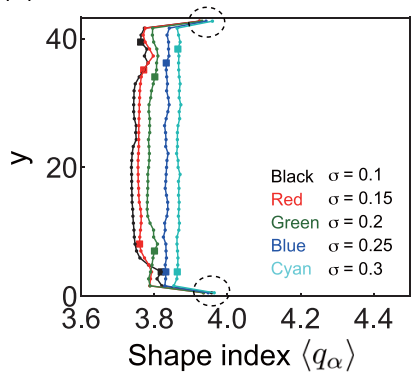

FIG. 8. (a) Examples of the flow profiles are shown $\left(N_{y}=\right.$ $\left.40, \lambda=0.01, p_{0}=3.33\right)$. The $N_{b}$ th and $N_{t}$ th layers, determined by the above procedure, are also shown with the square markers. (b) Spatial profiles of shape index $\left\langle q_{\alpha}\right\rangle$ are shown. The $N_{b}$ th and $N_{t}$ th layers are also shown with the square markers. The data points for the top and bottom layers are marked with dashed ellipsoids as a guide for eye. In (a) and (b), each data point corresponds to each layer.

simplifications were made in our model [see the paragraph below Eq. (B24)]. We have adopted those simplifications since they were not thought to affect the conclusion of our study.

\section{APPENDIX C: PREPARATION OF THE INITIAL CELLULAR CONFIGURATION}

We prepared the initial condition as shown in Fig. 6(a). In the initial condition, the cells in the bulk are set to regular hexagonal shape, while the cells at the boundaries, which are labeled with red dots in Fig. 6(a), are set to half of the regular hexagonal cells. The average cell area $\bar{A}$ is set to 1 . Then, we calculate the dynamics with $\sigma=0$ and $v_{\alpha}=0$ to relax the system for 50 time units, and then we set $\sigma$ and $v_{\alpha}$ to the target values. Figure $6(\mathrm{~b})$ is the configuration after the relaxation $\left(P_{0}=3.54\right)$.

\section{APPENDIX D: CONFIGURATION OBTAINED WHEN $\sigma=0$}

In Figs. 7(a) and 7(b), we show the tissue deformation observed without continuous cellular flow in the zero-noise limit $\sigma=0$ under a homogeneous cellular chiral torque and a gradient of cellular chiral torque, respectively.

\section{APPENDIX E: DEFINITION OF THE BOUNDARY LAYERS}

We systematically defined the boundary layers near either the bottom or the top boundaries. We discretized the area into $N_{y}$ layers from the 0th layer to the $\left(N_{y}-1\right)$ th layer. The $i$ th layer is defined as the layer ranging from $y=i L_{y} / N_{y}$ to $y=(i+1) L_{y} / N_{y}\left[i=0 \sim\left(N_{y}-1\right)\right]$. The boundary layers are defined as the 0 th $-N_{\mathrm{b}}$ th and $N_{\mathrm{t}}$ th $-\left(N_{y}-1\right)$ th layers, respectively, for those near the bottom and top boundaries.

(a)

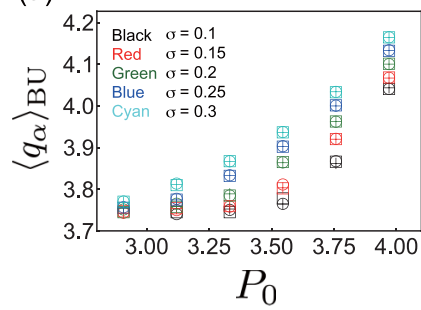

(b)

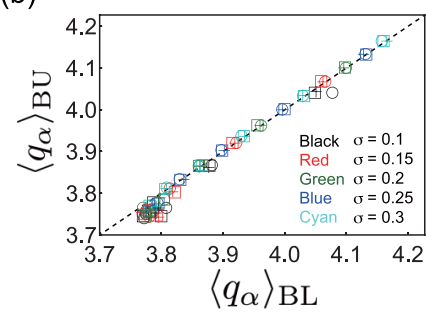

FIG. 9. (a) $\left\langle q_{\alpha}\right\rangle_{\mathrm{BU}}$ is plotted against $P_{0}$ for different values of $\sigma$ for a large system size $N_{y}=40$. The torque gradient $\lambda$ is fixed to 0.01 . The different symbols are for three different samples. (b) $\left\langle q_{\alpha}\right\rangle_{\mathrm{BU}}$ is plotted against $\left\langle q_{\alpha}\right\rangle_{\mathrm{BL}}$ for different $\sigma$ and $P_{0}$. The dashed line indicates $\left\langle q_{\alpha}\right\rangle_{\mathrm{BU}}=\left\langle q_{\alpha}\right\rangle_{\mathrm{BL}}$. The different symbols are for three different samples.

We calculated the average velocity of each layer by averaging the velocity of cells in the layer in time, and drew the velocity profiles as shown in Fig. 8(a) $\left(N_{y}=40, \lambda=\right.$ $\left.0.01, P_{0}=3.33\right)$. Using the velocity profile, we defined the boundary layers via the following procedure.

(1) We calculated the approximate bulk velocity $V_{\text {bulk }}^{\prime}$ and the approximate standard deviation $\sigma_{\text {bulk }}^{\prime}$ of the bulk velocity by averaging the velocity of the 15 th-24th layers.

(2) We smoothed the velocity profile to obtain $v=v^{\mathrm{sm}}(i)$ by a simple moving average, where the mean is taken from two data on either side of a central value.

(3) We determined $N_{\mathrm{b}}$ as the maximum integer $i<15$ which satisfies either $v^{\mathrm{sm}}(i)<V_{\text {bulk }}^{\prime}-3 \sigma_{\text {bulk }}^{\prime}$ or $v^{\mathrm{sm}}(i)>$ $V_{\text {bulk }}^{\prime}+3 \sigma_{\text {bulk }}^{\prime}$. Also, $N_{\mathrm{t}}$ is determined as the minimum integer $i>24$ which satisfies either $v^{\mathrm{sm}}(i)<V_{\text {bulk }}^{\prime}-3 \sigma_{\text {bulk }}^{\prime}$ or $v^{\mathrm{sm}}(i)>V_{\text {bulk }}^{\prime}+3 \sigma_{\text {bulk }}^{\prime}$.

In Fig. 8(a), we show the $N_{b}$ th and $N_{t}$ th layers, determined by the above procedure, with the square markers on examples of velocity profiles $\left(N_{y}=40, \lambda=0.01, P_{0}=3.33\right)$.

\section{APPENDIX F: RELATIONSHIP BETWEEN $\left\langle\boldsymbol{q}_{\alpha}\right\rangle_{\mathrm{BL}}$ AND $\left\langle\boldsymbol{q}_{\alpha}\right\rangle_{\mathrm{BU}}$}

In Fig. 8(b), we show examples of spatial profiles of the shape index $\left\langle q_{\alpha}\right\rangle$. We find that the shape index for the top and bottom layers is an outlier due to the flat boundaries which induce large cell deformation. To avoid the outliers, we eliminated the top and bottom layers when we calculated $\left\langle q_{\alpha}\right\rangle_{\mathrm{BL}}$.

In Fig. 9(a), we show the dependence of $\left\langle q_{\alpha}\right\rangle_{\mathrm{BU}}$ on the target shape index $p_{0}$ for the data in Fig. $5\left(N_{y}=40, \lambda=\right.$ $\left.0.01, P_{0}=3.33\right)$. We found that larger $\sigma$ and $p_{0}$ provide larger $\left\langle q_{\alpha}\right\rangle_{\mathrm{BU}}$. In Fig. 9(b), we show the relationship between $\left\langle q_{\alpha}\right\rangle_{\mathrm{BL}}$ and $\left\langle q_{\alpha}\right\rangle_{\mathrm{BU}}$ for the same data set. We find $\left\langle q_{\alpha}\right\rangle_{\mathrm{BU}} \approx$ $\left\langle q_{\alpha}\right\rangle_{\mathrm{BL}}$. As shown in Fig. 8(b), the spatial profiles of the shape index are nearly homogeneous except on the top and bottom layers. Hence, we obtain $\left\langle q_{\alpha}\right\rangle_{\mathrm{BU}} \approx\left\langle q_{\alpha}\right\rangle_{\mathrm{BL}}$.

[1] A. Mogilner and B. Fogelson, Cytoskeletal chirality: Swirling cells tell left from right, Curr. Biol. 25, R501 (2015).
[2] M. Inaki, J. Liu, and K. Matsuno, Cell chirality: Its origin and roles in left-right asymmetric development, Philos. Trans. R. Soc. B 371, 20150403 (2016). 
[3] S. R. Naganathan, T. C. Middelkoop, S. Fürthauer, and S. W. Grill, Actomyosin-driven left-right asymmetry: From molecular torques to chiral self organization, Curr. Opin. Cell Biol. 38, 24 (2016).

[4] L. Q. Wan, A. S. Chin, K. E. Worley, and P. Ray, Cell chirality: Emergence of asymmetry from cell culture, Philos. Trans. R. Soc. B 371, 20150413 (2016).

[5] N. Hirokawa, Y. Tanaka, and Y. Okada, Left-right determination: Involvement of molecular motor KIF3, cilia, and nodal flow, Cold Spring Harb. Perspect. Biol. 1, a000802 (2009).

[6] G. Lebreton, C. Géminard, F. Lapraz, S. Pyrpassopoulos, D. Cerezo, P. Spéder, E. M. Ostap, and S. Noselli, Molecular to organismal chirality is induced by the conserved myosin 1D, Science 362, 949 (2018).

[7] K. Taniguchi, R. Maeda, T. Ando, T. Okumura, N. Nakazawa, R. Hatori, M. Nakamura, S. Hozumi, H. Fujiwara, and K. Matsuno, Chirality in planar cell shape contributes to leftright asymmetric epithelial morphogenesis, Science 333, 339 (2011).

[8] M. Suzanne, A. G. Petzoldt, P. Spéder, J.-B. Coutelis, H. Steller, and S. Noselli, Coupling of apoptosis and L/R patterning controls stepwise organ looping, Curr. Biol. 20, 1773 (2010).

[9] K. Sato, T. Hiraiwa, E. Maekawa, A. Isomura, T. Shibata, and E. Kuranaga, Left-right asymmetric cell intercalation drives directional collective cell movement in epithelial morphogenesis, Nat. Commun. 6, 10074 (2015).

[10] C. Guillot and T. Lecuit, Mechanics of epithelial tissue homeostasis and morphogenesis, Science 340, 1185 (2013).

[11] T. Hiraiwa, E. Kuranaga, and T. Shibata, Wave propagation of junctional remodeling in collective cell movement of epithelial tissue: Numerical simulation study, Front. Cell Dev. Biol. 5, 66 (2017).

[12] K. Sato, T. Hiraiwa, and T. Shibata, Cell Chirality Induces Collective Cell Migration in Epithelial Sheets, Phys. Rev. Lett. 115, 188102 (2015)

[13] A. Tamada, S. Kawase, F. Murakami, and H. Kamiguchi, Autonomous right-screw rotation of growth cone filopodia drives neurite turning, J. Cell Biol. 188, 429 (2010).

[14] S. R. Naganathan, S. Fürthauer, M. Nishikawa, F. Jülicher, and S. W. Grill, Active torque generation by the actomyosin cell cortex drives left-right symmetry breaking, eLife 3, e04165 (2014).

[15] H. Yamanaka and S. Kondo, Rotating pigment cells exhibit an intrinsic chirality, Genes Cells 20, 29 (2015).

[16] Y. H. Tee, T. Shemesh, V. Thiagarajan, R. F. Hariadi, K. L. Anderson, C. Page, N. Volkmann, D. Hanein, S. Sivaramakrishnan, M. M. Kozlov, and A. D. Bershadsky, Cellular chirality arising from the self-organization of the actin cytoskeleton, Nat. Cell Biol. 17, 445 (2015).
[17] S. Fürthauer, M. Strempel, S. W. Grill, and F. Jülicher, Active chiral fluids, Eur. Phys. J. E 35, 89 (2012).

[18] S. Fürthauer, M. Strempel, S. W. Grill, and F. Jülicher, Active Chiral Processes in Thin Films, Phys. Rev. Lett. 110, 048103 (2013).

[19] S. Fürthauer, M. Neef, S. W. Grill, K. Kruse, and F. Jülicher, The Taylor-Couette motor: spontaneous flows of active polar fluids between two coaxial cylinders, New J. Phys. 14, 023001 (2012).

[20] T. Nagai and H. Honda, A dynamic cell model for the formation of epithelial tissues, Philos. Mag. B 81, 699 (2001).

[21] S. Curran, C. Strandkvist, J. Bathmann, M. de Gennes, A. Kabla, G. Salbreux, and B. Baum, Myosin II controls junction fluctuations to guide epithelial tissue ordering, Dev. Cell 43, P480 (2017).

[22] R. Farhadifar, J.-C. Röper, B. Aigouy, S. Eaton, and F. Jülicher, The influence of cell mechanics, cell-cell interactions, and proliferation on epithelial packing, Curr. Biol. 17, 2095 (2007).

[23] D. Bi, J. H. Lopez, J. M. Schwarz, and M. L. Manning, A density-independent rigidity transition in biological tissues, Nat. Phys. 11, 1074 (2015).

[24] See Supplemental Material at http://link.aps.org/supplemental/ 10.1103/PhysRevResearch.2.043326 for movies of cellular migration.

[25] J.-A. Park, J. H. Kim, D. Bi, J. A. Mitchel, N. T. Qazvini, K. Tantisira, C. Y. Park, M. McGill, S.-H. Kim, B. Gweon, J. Notbohm, R. Steward Jr, S. Burger, S. H. Randell, A. T. Kho, D. T. Tambe, C. Hardin, S. A. Shore, E. Israel, D. A. Weitz et al., Unjamming and cell shape in the asthmatic airway epithelium, Nat. Mater. 14, 1040 (2015).

[26] D. Bi, X. Yang, M. C. Marchetti, and M. L. Manning, MotilityDriven Glass and Jamming Transitions in Biological Tissues, Phys. Rev. X 6, 021011 (2016).

[27] S. Etienne-Manneville and A. Hall, Rho GTPases in cell biology, Nature (London) 420, 629 (2002).

[28] D. Gilmour, M. Rembold, and M. Leptin, From morphogen to morphogenesis and back, Nature (London) 541, 311 (2017).

[29] P. Ray, A. S. Chin, K. E. Worley, J. Fan, G. Kaur, M. Wu, and L.Q. Wan, Intrinsic cellular chirality regulates left-right symmetry breaking during cardiac looping, Proc. Natl. Acad. Sci. USA 115, E11568 (2018).

[30] Z. Sun, C. Amourda, M. Shagirov, Y. Hara, T. E. Saunders, and Y. Toyama, Basolateral protrusion and apical contraction cooperatively drive Drosophila germ-band extension, Nat. Cell Biol. 19, 375 (2017)

[31] M. Williams, W. Yen, X. Lu, and A. Sutherland, Distinct apical and basolateral mechanisms drive planar cell polaritydependent convergent extension of the mouse neural plate, Dev. Cell 29, 34 (2014). 